\title{
A Simulation Environment for the Design of Advanced Chiller Control Systems
}

\author{
Michele Albieri ${ }^{1}$, Alessandro Beghi², Cristian Bodo ${ }^{2}$, Luca Cecchinato ${ }^{3}$ \\ ${ }^{1}$ Rhoss S.p.A., via Oltre Ferrovia, I-33033 Codroipo, Italy, michele.albieri@rhoss.com \\ 2 Dipartimento di Ingegneria dell'Informazione, Università di Padova, via Gradenigo 6/B, I- \\ 35131 Padova, Italy, beghi@dei.unipd.it \\ ${ }^{3}$ Dipartimento di Fisica Tecnica, Università di Padova, via Venezia 1, I-35131 Padova, \\ Italy, ceck@unipd.it
}

\begin{abstract}
In this paper we address the problem of designing advanced control systems for increasing the performances of one of the key elements of an HVAC system, the chiller unit. In particular, we present a simulation environment based on Matlab/Simulink that has been validated on a state-of-the-art experimental facility and used to design an adaptive controller for single scroll compressor, packaged air-cooled water chillers, that allows to substantially increase the energy performance of the system, as well as to achieve excellent regulation performances in process applications.
\end{abstract}

\section{Introduction}

Efficient use of energy is one of the main strategic measures not only for the conservation of fossil energy resources but also for abatement of air pollution and the slowing down of anthropogenic climate change. The requirement of primary energy to cool and to heat buildings is an important part of the overall energy consumption in Western countries, summing up to about $30 \%$ of the U.S. and European global energy consumption, and reaching even higher percentages in country such as Italy (up to 50\%), due to the increasing use of air conditioning units for cooling residential and office buildings during summer. In fact, in the last years split -system air conditioners are being increasingly installed for cooling residential buildings, offices, and shops during the summer period. Such devices are clearly less expensive than other HVAC (Heating Ventilation and Air Conditioning) solutions, but they often lack in efficiency; as a direct consequence, the derived pollution effects on the environment are steadily increasing. These facts motivated the European Commission to deliberate on the energy performance of buildings (EPBD), with the Directive 2002/91/EC which imposes several actions to achieve prudent and rational use of energy resources and to reduce the environmental impact of the energy use in buildings. This can be accomplished by increasing both the energy performance of new and existing buildings and the efficiency of cooling/heating systems. It is generally agreed that in spite of the advancements made in computer technology and its impact on the development of new control methodologies for HVAC systems aiming at improving their energy efficiencies, the process of operating HVAC equipment in commercial and industrial buildings is still a low-efficient and high-energy consumption process $[1,2,3,4]$. Classical HVAC control techniques such as ON/OFF controllers (thermostats) and proportional-integral-derivative (PID) controllers are still very popular, due to their low cost and ease of tuning and operation. However, these simple controllers do not grant a sufficient energy efficiency and therefore plants operating with such control architectures 
on which the model has to be validated The heat load is transferred to the hydraulic section by a plate heat exchanger (Plate HX in Figure 1).

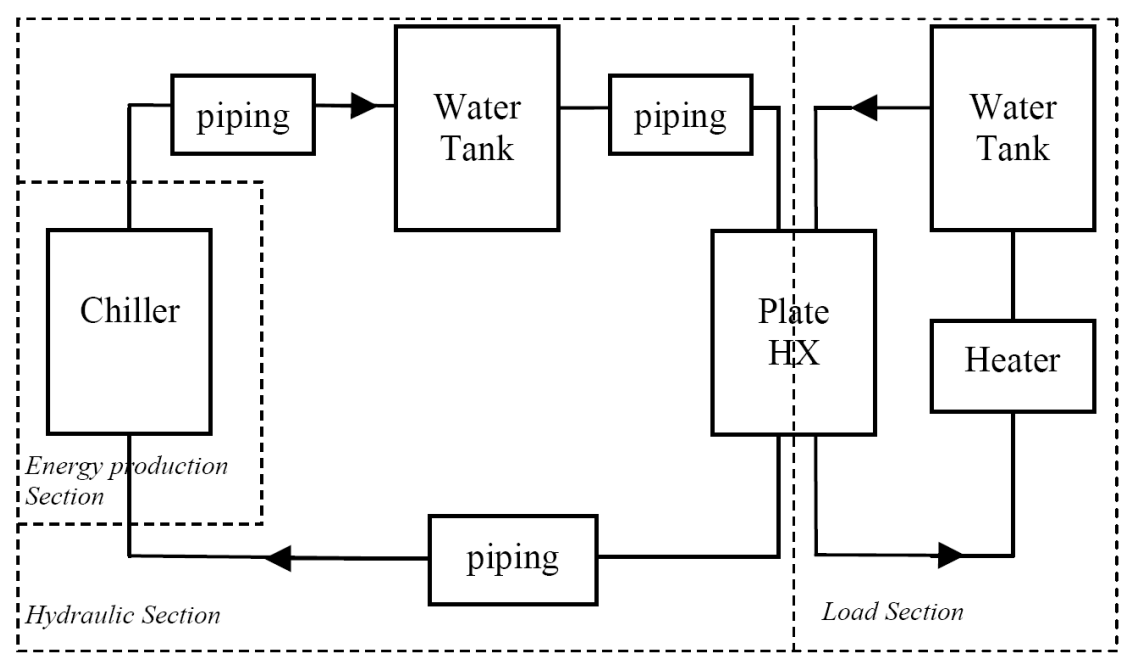

Figure 1: System block structure

The thermal behaviour of such a plant can be usefully analyzed by a lumped formulation of the conservation equations. The elements of the plant are simulated through blocks, and the heat transfer processes are considered as concentrated inside the blocks. Furthermore, the following hypotheses are introduced:

- The water thermal properties are considered constant;

- The water is considered incompressible;

- The three sections have constant water mass flow;

- There is no mass accumulation inside blocks;

- $\quad$ piping and water tanks are considered adiabatic.

The system dynamics are governed by the mass, momentum, and energy conservation laws. The mass and energy equations are implemented as block equations for each component of the plant, where each block is modelled as thermodynamic open system. The dynamic behaviour of the plant is thus obtained solving the fluid flow problem and the energy problem. No solution of the momentum equation is needed because of the constant water mass flow assumption in the three sections, that grants that pressure losses are constant inside each block. Thus the fluid flow problem consists only in the determination of the mass flow rate and the equations for the $k$-th block may be simply written as follows:

$m_{k, i}-m_{k, o}^{k}=0$,

where dependence on the time variable $\tau$ is omitted for notational convenience, if possible. The thermal problem consists in the determination of the temperature values at the outlet of the $k$-th block. The energy equation at time $\tau$ can be written as follows:

$$
\|_{k, i} \cdot\left(c_{p} T_{k, i}+e_{p, k, i}+e_{c, k, i}\right)-\phi_{k, o} \cdot\left(c_{p} T_{k, o}+e_{p, k, o}+e_{c, k, o}\right)+\frac{\partial}{\partial \tau} \int_{0}^{v_{k}} \rho e d v=\frac{d Q_{k}}{d \tau}-\frac{d L_{k}}{d \tau}
$$

Since the model has to be used for control system design, it is not possible to proceed under the well-mixed hypothesis for the water inside the system components. In fact, the water content and the dynamics of the water tanks strongly influence the behaviour of the 
chiller control system. Therefore, each water tank is modelled as two separate parts connected in series (see Figure 2). In the first part a well-mixed condition is assumed, while in the second part a perfect stratification condition is considered. For the well-mixed section, with the above mentioned simplifying hypotheses and neglecting kinetic and potential energy variations, equation (2) at time $\tau$ becomes:

$n \&_{k, i} c_{p} T_{k, i}-n \&_{k, o} c_{p} T_{k, f}+f_{k} \rho V_{k} c_{p} \frac{d T_{k, f}}{d \tau}=0$,

where $f_{k}$ is the well-mixed section fraction of the tank total volume. This parameter is given as a function of a cylindrical tank geometric dimension and of the water velocity by means of FVM (Finite Volume Method) three-dimensional simulations.

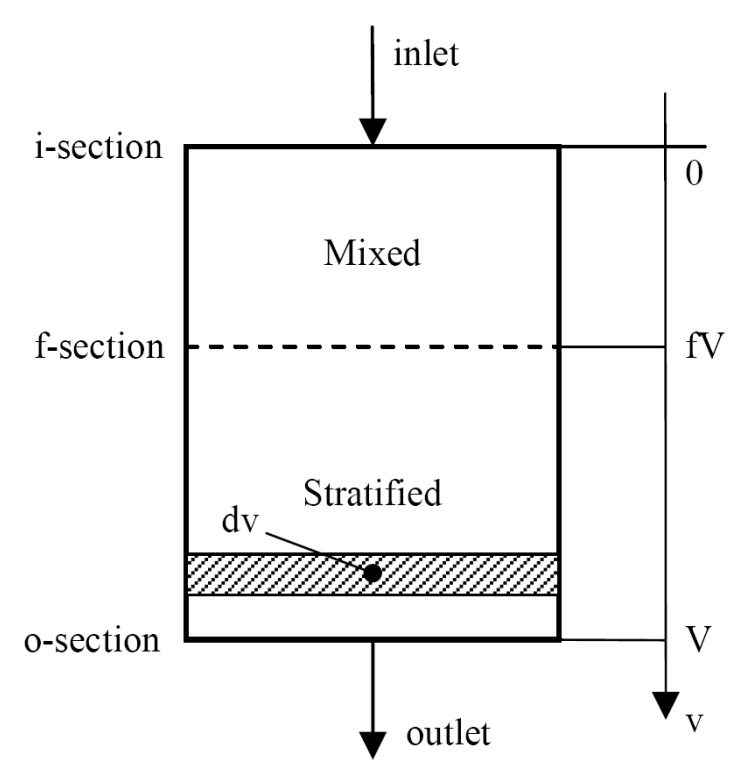

Figure 2: Water tank and piping scheme

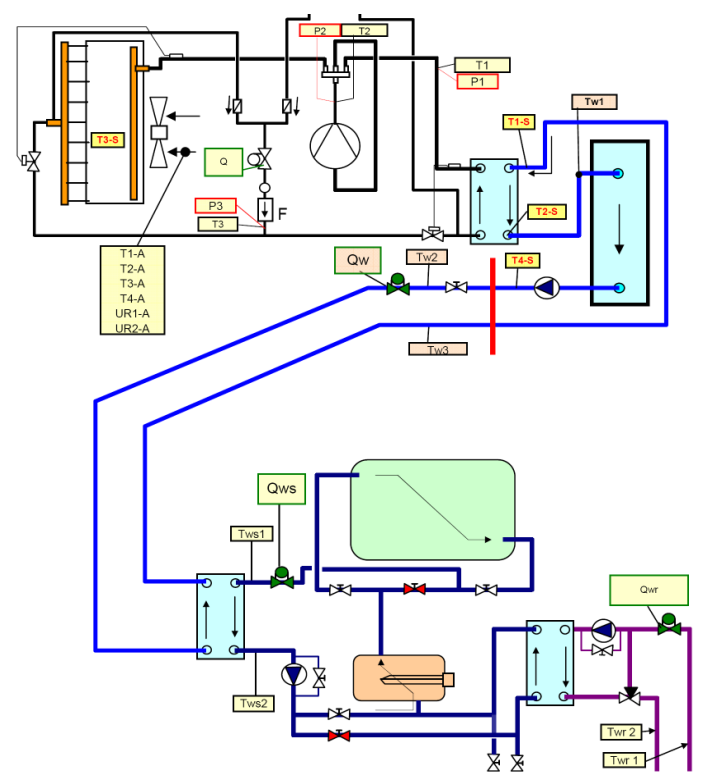

Figure 3: System test facility

For the stratified section of the water tank, the temperature in each infinitesimal volume $d v$ depends only on the inlet time $t$ of the associated infinitesimal water mass at the integration time $\tau$, and equation (3) becomes:

$\operatorname{lof}_{k, i} C_{p} T_{k, f}(\tau)-n_{k, o}^{\&} C_{p} T_{k, o}(\tau)+\frac{\partial}{\partial \tau} \int_{T_{k} V}^{v_{k}} \rho c_{\rho} T_{k, i}(t) d v=0$.

Since no mixing occurs inside the stratified section of the water tank, the volumetric coordinate $v$ associated to the position of an infinitesimal water mass inside the tank can be expressed as a function of the time instant $t$ when the water mass entered the stratified portion of the tank. The resulting expression for $v$ with reference to the actual integration time $\tau$ :

$v=A_{k} x=f_{k} V_{k}+(\tau-t) \frac{\left(1-f_{k}\right) V_{k}}{t_{c, k}}$, 
where $t_{c}$ is the tank section time constant defined as:

$t_{c, k}=\left(1-f_{k}\right) \frac{\rho V_{k}}{n_{\phi_{k, i}}^{k}}$

Differentiating (5) and substituting in (4), the following equation is obtained:

$n \phi_{k, i} c_{p} T_{k, f}(\tau)-n \phi_{k, o} c_{p} T_{k, o}(\tau)+\frac{\partial}{\partial \tau} \int_{\tau}^{\tau-t_{c}}-\rho c_{p} \frac{\left(1-f_{k}\right) V_{k}}{t_{c, k}} T_{k, f}(t) d t=0$

and integrating (6), the final equation for the stratified section is determined:

$\&_{k, o} c_{p} T_{k, o}(\tau)=m_{k, i}^{\&} c_{p} T_{k, f}\left(\tau-t_{c}\right)$

Combining (7) and (3) at each time step, the water tank block energy equation is solved and the outlet temperature is determined. The two equations can also be merged using Laplace transforms, thus obtaining the following first-order transfer function for the tank:

$W_{k}(s)=\frac{T_{k, o}(s)}{T_{k, i}(s)}=\frac{e^{-s t_{c}}}{1-s \frac{f_{k} \rho V_{k}}{n_{k, i}^{\&}}}$

The same approach is used to model piping blocks, although the FVM analysis indicated that in this case water mixing is negligible. The resulting simplified model for piping and tanks, derived under the above mentioned hypotheses, have been analyzed and validated by means of tests performed on the experimental facility, as described in Section 3.

Taking into account that the chiller and heater water content is negligible, the energy equation for these two blocks at time $\tau$ becomes:

$$
n \|_{k, i} c_{p} T_{k, i}-n \&_{k, o} c_{p} T_{k, o}=\frac{d Q_{k}}{d \tau}
$$

where for the chiller, the RHS term, as well as the electrical absorbed power, is determined on the basis of data provided by the manufacturer as a function of water inlet temperature, mass flow, and external air temperature. Thus, the dynamic phenomena associated with heat and mass transfer, especially during start-ups, are neglected. Different control algorithms can be implemented to simulate chiller operation (see also Section 2.2.)

For the plate heat exchanger block, equation (9) is used for both the load and the hydraulic section. The RHS term in (9) is determined as a function of inlet temperatures defining a heat exchanger efficiency $\varepsilon$ and applying the $\varepsilon$-NTU method for heat exchangers:

$$
\frac{d Q_{k}}{d \tau}=\varepsilon \cdot \min \left(n_{k, i, H}, n_{k, i, L}^{\&_{k}}\right) \cdot c_{p}\left|T_{k, i, H}-T_{k, i, L}\right|
$$

The final system of non-linear equations obtained from equations (1) and (2) of each block and from equation (10) is integrated in the Matlab/Simulink simulation environment. 


\subsection{Chiller control}

Typically, a chiller without capacity control can be regulated in two different ways, namely by controlling the chiller evaporator water outlet or the chiller evaporator water inlet. In both cases the compressor is switched on when the controlled temperature reaches a given upper boundary value whereas it is switched off at a lower boundary value. The difference between the two values is called water temperature differential, and its value clearly affects the width of the oscillations of the supply water temperature as well as the number of start-ups of the compressor. A low value of the water temperature differential allows to obtain a more constant water temperature, but it also causes an increase of the number of compressor start-ups, which cannot be larger of a given limit value, fixed by the constructor. Also, the value of differential cannot be decreased arbitrarily, but there is a lower limit value which depends on the plant water content.

While both control strategies maintain constant the user water supply temperature in full load conditions, outlet water temperature control grants better performance in chiller part load conditions since it maintains mean water supply temperature fairly constant during on/off operations. The performance of such control scheme can be further improved by implementing advanced strategies aimed at increasing the energy efficiency of the chiller and/or its accuracy in maintaining a given set point value of the supply temperature. The chiller-plant model described above has been used to design such an advanced controller, named AdaptiveFunction Plus controller, which is presently patent pending and will be better detailed in forthcoming publications. Here we mention only its main characteristics, that are the following:

1) Low energy consumption: the Economy function combines comfort with low energy consumption. This is achieved by adjusting the set-point value and optimizing compressor efficiency on the basis of the actual load conditions. It is thus possible to achieve significant seasonal energy savings compared to water chillers and heat pumps of an equivalent power with traditional control logic [5], [6].

2) High precision: with the Precision function, it is possible to achieve as little oscillation as possible at part load conditions, both in terms of the average set-point water temperature delivered to the users and of standard deviation from the set-point temperature. Thanks to a special Virtual Tank function, the controller can work well also in systems with a low water content of down to 2 litres $/ \mathrm{kW}$ cooling capacity, even without the presence of a water tank.

3) Estimation of the system thermal inertia: during the system first start-up the Autotuning function can estimate the characteristics of the thermal inertia and system dynamics in order to identify the optimal value of the control parameters. This function is always active and makes it possible to adapt control parameters quickly to changes in the water circuit and thus in the system water contents.

\section{Simulation model validation}

\subsection{The test facility}

In Figure 3 the experimental test facility used to validate the simulation model is schematically shown. The energy production section is equipped with a Rhoss TCAEY 130 packaged air-cooled water chiller with R410A refrigerant, cooling capacity of $26.79 \mathrm{~kW}$ and EER (Energy Efficiency Rating) of 2.44 in the following operating conditions: condenser input air temperature $35{ }^{\circ} \mathrm{C}$; chilled water temperature $7{ }^{\circ} \mathrm{C}$; temperature differential at evaporator $5{ }^{\circ} \mathrm{C}$. The chiller is equipped with a single scroll compressor without capacity 
control. The hydraulic section has a 45 litres water tank and a piping total volume of 36 litres. The pump constant water flow rate is $1.28 \mathrm{~kg} / \mathrm{s}$. The load section has an electrical heater, with a heating capacity in the range $0-50 \mathrm{~kW}$ and a 480 litres water tank. A brazed plate heat exchanger (BHE) of 0.9 efficiency is installed.

Thermocouples and pressure transducers are placed as shown in Figure 3. Water temperatures are measured with Pt100 thermometers placed inside mixing chambers at the inlet and outlet of each heat exchanger. The R410A temperatures are measured with Pt100 thermometers placed on the pipe wall. A $\pm 0.3^{\circ} \mathrm{C}$ accuracy is estimated for all the temperature measurements. The R410A mass flow rate is measured by a Coriolis mass flow meter placed upstream of the throttling valve. The claimed accuracy is $\pm 0.1 \%$ of reading. Water volumetric flow rates are measured by electromagnetic meters (accuracy $\pm 0.2 \%$ of reading). The $\mathrm{R} 410 \mathrm{~A}$ pressures are recorded with strain-gauge transducers at the suction and outlet the compressor. The accuracy is $\pm 10 \mathrm{kPa}$ according to the calibration report from the manufacturer. Electrical absorbed power is recorded with an electronic transducer (with an accuracy $\pm 0.5 \%$ of the reading value).

Tests have been carried out with the condenser positioned in a climatic room maintained at $35{ }^{\circ} \mathrm{C}$ air temperature. By controlling the heater thermal power, the chiller has been tested in full load conditions and at 25\%, 50\%, 75\% part load conditions. The mean systems efficiencies in terms of EER have been obtained by integrating the power absorption and the cooling capacity, computed from the instantaneous values of refrigerant mass-flow, condenser outlet and evaporator outlet enthalpies, which are computed from pressure and temperature values on the basis of refrigerant properties as represented in the NIST Reference Fluid Thermodynamic and Transport Properties REFPROP, Version 7.0 [7]. The computed cooling capacity on the refrigerant side has been compared and validated with the computed capacity on the water side.

\subsection{Validation test campaign}

A wide validation test campaign for the developed simulation environment has been carried out on the test facility described in Section 3.1. Tests have been performed on the chiller with inlet water temperature control, for different values of the electrical heat load, condenser supply air temperature, water mass flow, set-point and differential. The system dynamics and energy performances obtained from experimental tests have been compared to those obtained in the virtual simulation environment. As an example, in Figures 4 and 5, real and virtual absorbed power, chiller and water tank outlet temperatures are compared at $20 \%$ and $75 \%$ part load ratio. It is worth noticing that the chiller cooling capacity and absorbed power model are based on manufacture data which do not consider energy losses during compressor start-ups. As a consequence, the expected absorbed power is not exactly predicted, especially during the first period of the compressor working cycle.

From Figure 5 it can be observed that the simplified, monodimensional model for the water tank is only partially in agreement with the experimental data, as expected, in particular at high thermal load. However, it is fully adequate for the purpose of controller design reproducing the main dynamic behaviours that are relevant for controller design.

\section{Development and performances of the adaptive algorithm}

\subsection{Virtual development and prototyping}

The fully validated simulation environment of the chiller plant system has been extensively 
used to design the AdaptiveFunction Plus controller. In fact, the simulation environment is able to reproduce all the system transient and steady state behaviours that are crucial to assess the performance of the control system. In the following, the performance of the AdaptiveFunction Plus control algorithm are illustrated by reporting the time behaviour of some key quantities, as well as an indication of its energy performance given in terms of energy efficiency rating (EER).

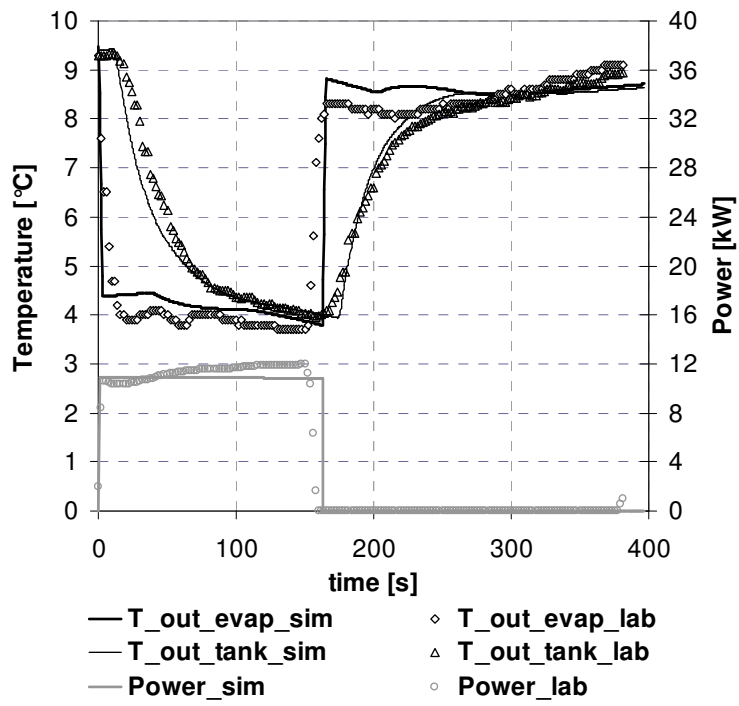

Figure 4: Comparison between experimental and virtual system at $20 \%$ part load ratio.

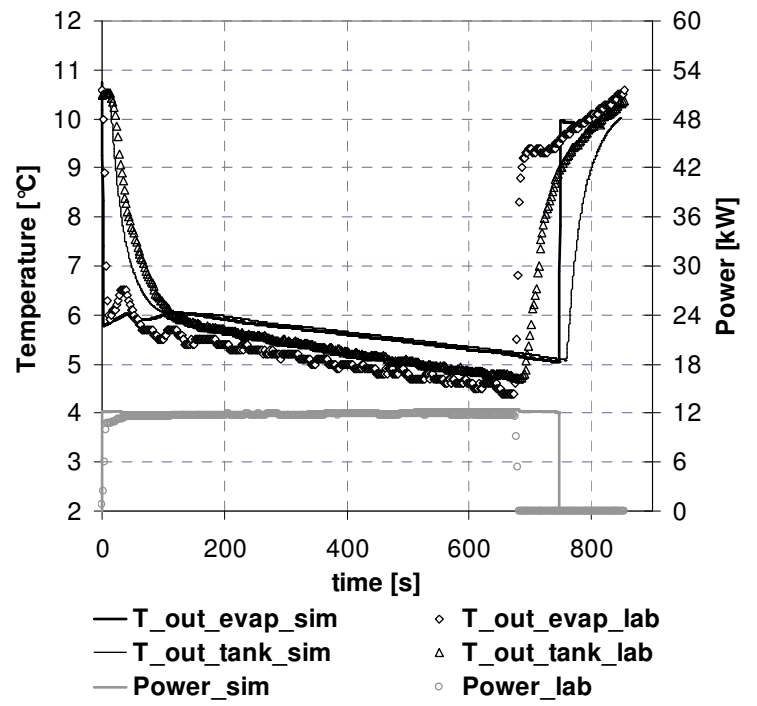

Figure 5: Comparison between experimental and virtual system at $75 \%$ part load ratio.

As outlined in Section 2.2, the adaptive controller Precision function minimizes the water outlet temperature oscillation by appropriately changing the water differential, and thus the upper and lower boundary temperature, as a function of the part load ratio.

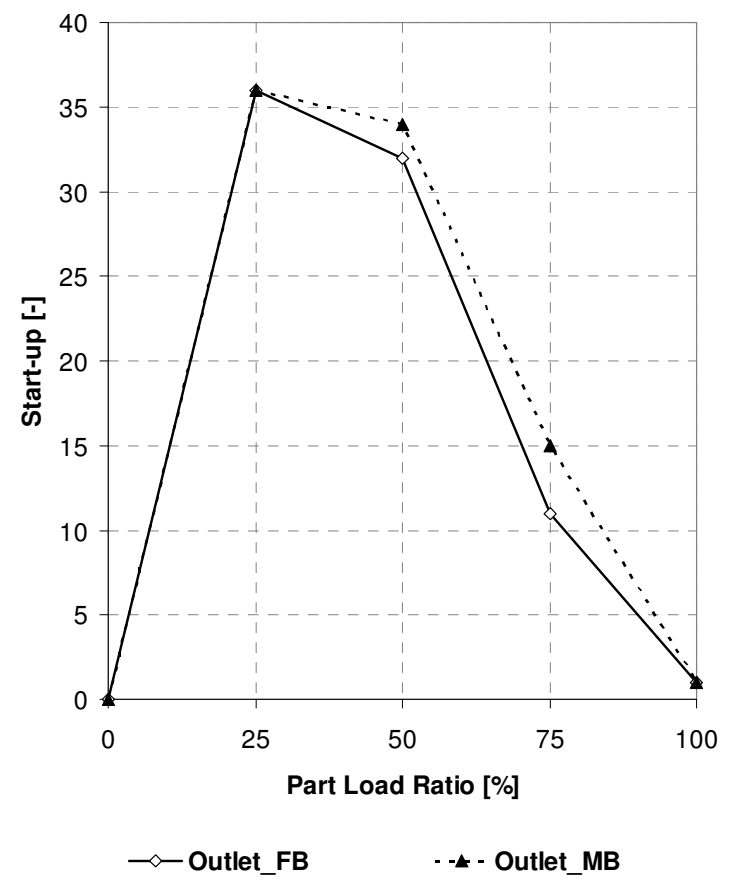

Figure 6: Number of start-ups for different controllers during four hour cycling. operation.

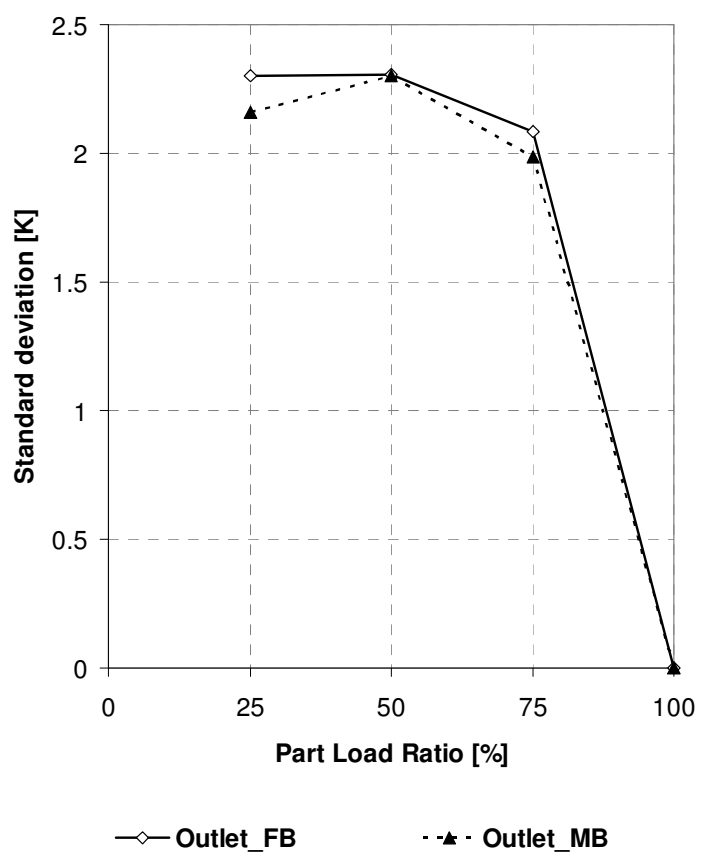

Figure 7: Chiller water outlet standard deviation from set-point temperature for different controllers. 


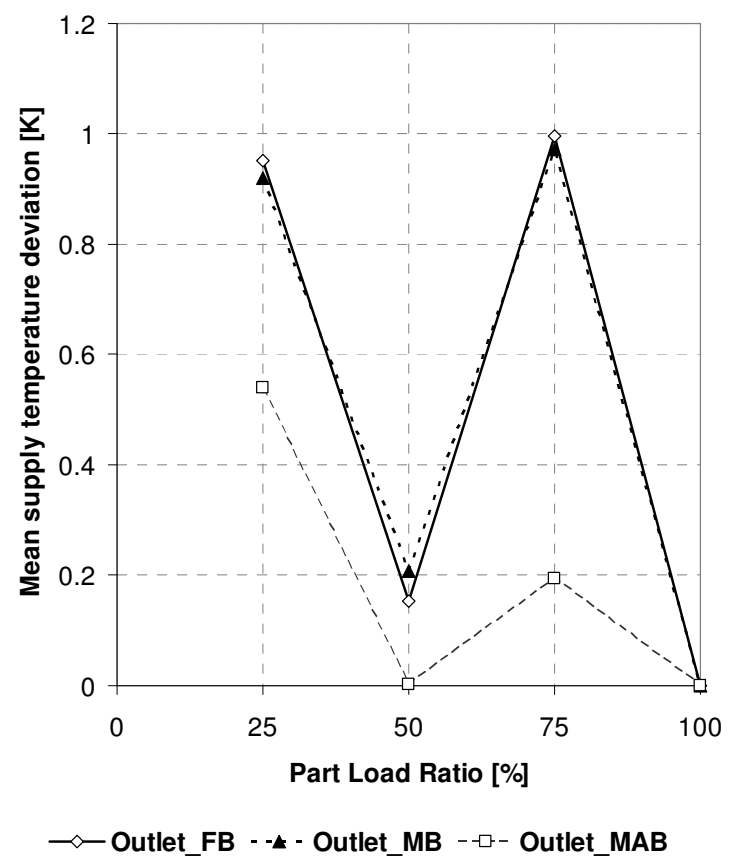

Figure 8: Difference between average (on+off period) chiller water outlet temperature and set-point temperature for different controllers.

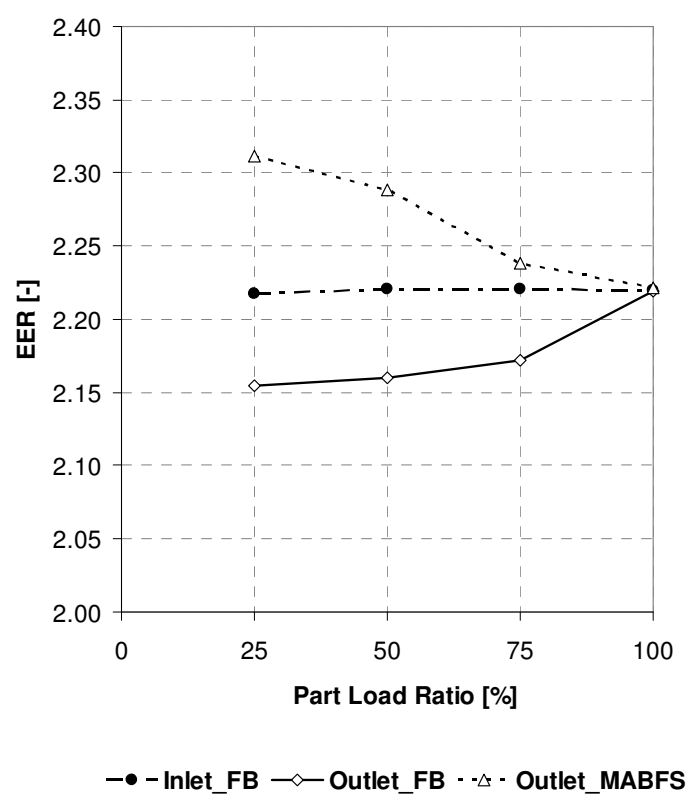

Figure 10: Chiller Energy Efficiency Rating for different controllers.

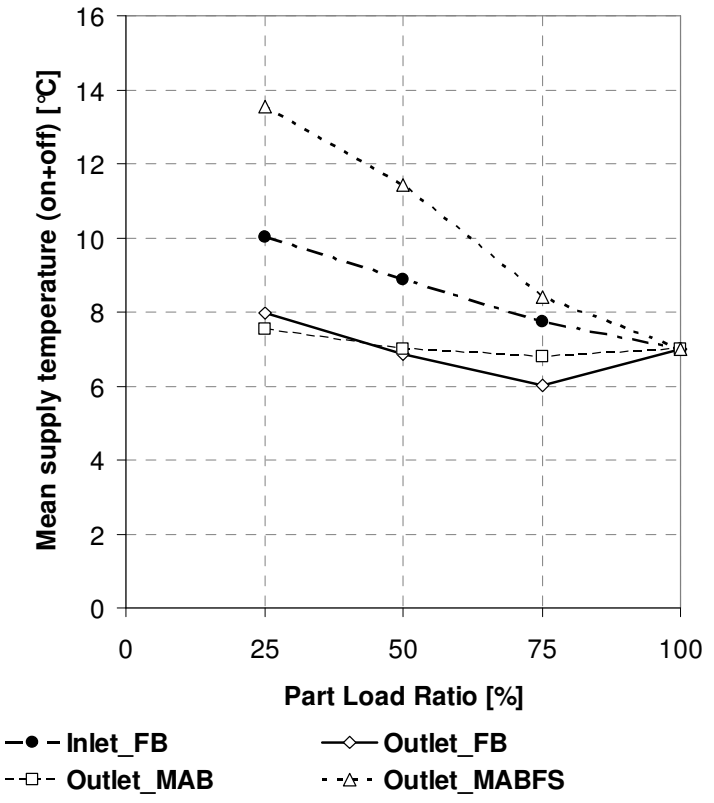

Figure 9: Average (on+off period) chiller water outlet temperature for different controllers.

In Figures 6 and 7, the number of start-ups and the standard deviation from the outlet water set-point $\left(7^{\circ} \mathrm{C}\right)$ is plotted for a standard outlet water temperature control (Outlet_FB) with $4.8{ }^{\circ} \mathrm{C}$ differential and for the adaptive control which uses a moving boundary logic (Outlet_MB). Comparing the results, it is clear that the developed control allows a greater precision, reducing standard deviation and increasing the number of start-up in particular for values of the part load ratio between $25 \%$ and $75 \%$. An improved version of the control allows an asymmetrical positioning of the two boundaries with reference to the set-point temperature (Outlet_MAB). In Figure 8, the difference between the average outlet water temperature during an on-off cycle and the set point temperature of the previous system is compared to this improved control. The moving asymmetrical boundary algorithm appears to be extremely accurate

up to $50 \%$ part load ratio, below this value the controller antifreeze function reduces its performance.

The adaptive controller Economy function improves the system energy efficiency by adjusting the outlet water set-point value on the basis of the actual load conditions. If the 
part load ratio decreases, the control rises the set-point thus increasing the efficiency rating of the chiller. In Figures 9 and 10, the floating set-point control algorithm (Outlet_MABFS) is compared with both an outlet and inlet water temperature control in terms of average outlet water temperature and EER. It is worth noting that for part load ratios lower than $75 \%$, the EER improvement with respect to the outlet water temperature control varies from $7.3 \%$ to $3.0 \%$.

\subsection{Seasonal energy efficiency rating}

Finally, the developed algorithm has been then implemented on board of a commercial chiller unit, and its performance has been evaluated on the experimental testing facility. AdaptiveFunction Plus Algorithm real energy performance has been evaluated in terms of seasonal energy efficiency obtained for the load, air temperature and energy weighting coefficients used in the calculation of the ESEER (European Seasonal Energy Efficiency Rating) [8]. In Table 2 the energy efficiency ratings EER for the four test conditions indicated in the calculation methodology and the seasonal energy efficiencies obtained for the inlet control, the moving asymmetrical boundary algorithm and the floating set-point control are reported. The floating set-point control algorithm seasonal energy rating improvement with respect to the adaptive outlet water temperature control and to the standard inlet control is $9.1 \%$ and $6.2 \%$ respectively.

Table 2: Seasonal energy rating for different control algorithms.

\begin{tabular}{|c|c|c|c|c|c|c|}
\hline \multirow[b]{2}{*}{ Part Load Ratio [\%] } & \multicolumn{4}{|c|}{ EER } & \multirow[t]{2}{*}{ ESEER } & \multirow[t]{2}{*}{$\Delta[\%]$} \\
\hline & 25 & 50 & 75 & 100 & & \\
\hline Ambient temperature $\left[{ }^{\circ} \mathrm{C}\right]$ & 20 & 25 & 30 & 35 & & \\
\hline Weighting coefficients [\%] & 23 & 41 & 33 & 3 & & \\
\hline Inlet_FB & 3.06 & 2.88 & 2.57 & 2.23 & 2.80 & 2.8 \\
\hline Outlet_FB & 2.94 & 2.79 & 2.54 & 2.23 & 2.72 & - \\
\hline Outlet MABFS & 3.28 & 3.09 & 2.69 & 2.23 & 2.97 & 9.1 \\
\hline
\end{tabular}

\section{Conclusions}

In this paper we addressed the problem of deriving a simulation environment for the design of advanced chiller control systems. The novelty of the adopted approach with respect to existing ones is that all of the dynamic behaviours that are relevant for controller design have been taken into account (see, e.g., the modelling of water tanks), while neglecting or simplifying other dynamic phenomena that contribute only marginally to the assessment of the overall controller-plant performance.

The model has been satisfactorily validated in a state-of-the-art experimental facility, by performing an extensive validation campaign. The environment has been used to design a novel control algorithm for single scroll compressor, packaged air-cooled water chillers, which allows to increase both control accuracy and energy performance. It is worth noticing that the availability of a fully validated virtual environment allows to obtain an assessment of the system performance by means of virtual tests that would be very difficult, if not impossible, to be performed on experimental test facilities or commercial plants. This is fully in agreement with the experience in other control engineering applications, such as the automotive field, where the use of virtual protoyping tools is nowadays a common practice. 


\section{REFERENCES}

[1] B. Arguello-Serrano and M. Velez-Reyes, "Nonlinear Control of a Heating, Ventilating, and Air Conditioning System with Thermal Load Estimation," IEEE Trans. Contr. Sys. Tech., vol. 7. no. 1, pp. 56-63, 1999.

[2] Z. Huguang and L. Cai, "Decentralized nonlinear adaptive control of an HVAC system." IEEE Trans. Sys. Man. Cyb. Part C: Appl. and Rev., vol. 32, no. 4, pp. 493-498, 2002.

[3] R. Shoureshi,"Intelligent control systems: Are they real, J. Dynamic Syst., Measur. Contr., vol. 115, pp. 15-19, Jan. 1993.

[4] M. Yaqub and S. M. Zubair, "Capacity control for refrigeration and air conditioning systems: A comparative study, Int. J. Energy Res. Tech., vol. 123, pp. 9-99, Apr. 2001.

[5] J.E.Braun, S.A. Klein, J.W. Mitchell and W.A. Beckman, "Methodologies for optimal control to chilled water systems without storage, ASHRAE Transactions, vol. 95(1), 1989.

[6] E. Fornasieri, "Refrigeratori d'acqua con compressore volumetrico: come promuovere l'efficienza energetica", $43^{\circ}$ Convegno Internazionale AICARR, vol. 3 , pp. 17-46, Milano 78 Mar. 2002.

[7] E.W. Lemmon, M.O. McLinden, and M.L. Huber,"NIST Reference Fluid Thermodynamic and Transport Properties Refprop 7.0, NIST Std. Database, 2002.

[8] AA.VV., "Energy Efficiency and Certification of Central Air Conditioners (EECCAC), Ed:Armines, vol. 3, April 2003. 